\title{
Growth differentiation factor 9 and cumulus cell supplementation in in vitro maturation culture media enhances the viability of human blastocysts
}

\author{
Mahla Honari Chatroudi', Mohammad Ali Khalilii ${ }^{1}$, Sareh Ashourzadeh ${ }^{3}$, Fatemeh Anbari ${ }^{2}$, Abbas Shahedi ${ }^{1}$, Somayyeh Safari ${ }^{4}$ \\ 'Department of Anatomy and Cell Biology, Shahid Sadoughi University of Medical Sciences and Health Services, Yazd; ${ }^{2}$ Department of Reproductive \\ Biology, Yazd Reproductive Science Institute, Shahid Sadoughi University of Medical Sciences, Yazd; ${ }^{3}$ Kerman Infertility Center, Afzalipour Hospital, \\ Kerman University of Medical Sciences, Kerman; ${ }^{4}$ Hospital Research Development Committee, Nekoei-Hedayati-Forghani Hospital, Qom University of \\ Medical Science, Qom, Iran
}

Objective: In vitro maturation (IVM) of immature oocytes can be useful for some infertile patients. In IVM programs, the rates of embryo formation and pregnancy are low. Therefore, it is essential to recognize the main factors involved in regulating oocyte maturation in vitro. The purpose of this study was to investigate the effects of growth differentiation factor 9 (GDF9) and cumulus cell (CC) supplementation in IVM medium on the rates of embryo formation and viability of human blastocysts.

Methods: A total of 80 germinal vesicle oocytes from stimulated cycles underwent an IVM program. The oocytes were divided into four groups, where group I consisted of IVM media only and served as the control, group II consisted of IVM+CCs, group III consisted of IVM+GDF9 $(200 \mathrm{ng} / \mathrm{mL})$, and group IV consisted of IVM+CCs+GDF9 $(200 \mathrm{ng} / \mathrm{mL})$. Intracytoplasmic sperm injection was performed on the IVM oocytes, and the cleavage embryos that were generated were vitrified. Following thawing, the embryos were cultured for 3 additional days, and the viability rates of the developed blastocysts were determined.

Results: The maturation rate of the oocytes did not differ significantly across the four groups. The fertilization rate in group II was significantly higher than that in the control group ( $76.5 \%$ vs. $46.2 \%)$. Embryo formation was significantly more frequent in all experimental groups than in the control group, while blastocyst formation did not show significant differences in the three experimental groups compared to the control. The mean viability rates in groups II, III, and IV were $58.16 \%, 55.91 \%$, and $55.95 \%$, respectively, versus $37.78 \%$ in the control group $(p<0.05)$. Conclusion: Supplementation of IVM culture media with GDF9 and CCs enhanced the fertilization, embryo formation, and viability rates of blastocysts generated from vitrified cleavage embryos.

Keywords: Cumulus cells; Embryo formation; Growth differentiation factor 9; In vitro maturation

Received: July 3, 2019 · Revised: July 29, 2019 · Accepted: August 26, 2019 Corresponding author: Mohammad Ali Khalili

Department of Anatomy and Cell Biology, Shahid Sadoughi University of Medical Sciences and Health Services, Bouali Ave, Safaieh City, Yazd 8916877391, Iran Tel: +98-35-38247087 Fax:+98-35-8247085-6 E-mail:Khalili59@hotmail.com

*This study was financially supported by the Shahid Sadoughi University of Medical Sciences, Yazd, Iran.

This is an Open Access article distributed under the terms of the Creative Commons Attribution Non-Commercial License (http://creativecommons.org/licenses/by-nc/4.0/) which permits unrestricted non-commercial use, distribution, and reproduction in any medium, provided the original work is properly cited.

\section{Introduction}

In vitro maturation (IVM) of oocytes is a promising strategy in assisted reproductive technology. In controlled ovarian hyperstimulation $(\mathrm{COH}), 15 \%$ of oocytes are immature at the germinal vesicle (GV) or metaphase I (MI) stages. Rescue IVM of these immature oocytes can increase the chances of embryo access, especially in poor responders $[1,2]$. In addition, the IVM technique plays a highly important role in the elimination of ovarian hyperstimulation syndrome, which is a 
possible side effect of gonadotropin stimulation in patients with polycystic ovary syndrome (PCOS) [3,4]. Oocytes have the ability to influence gene expression in cumulus cells (CCs) and mural cells, even in the presence of follicle-stimulating hormone (FSH), which indicates that the intracellular behavior of CCs is affected by their close relationship with the oocyte [5].

Although the quality of the oocyte largely determines the quality of the generated embryo, changes in the microenvironment of in vitro culture also affects embryonic development $[6,7]$. In recent years, advances have been made in using $\mathrm{CCs}$ and growth factors to enhance the developmental competence of oocytes [8]. Zhu et al. [9] showed that co-culture of immature oocytes with CCs increased the possibility of polar body exclusion to $10 \%$ within 24 hours. However, they reported that the rate of embryo formation in these oocytes was still low. Previous studies have attempted to assess the effects of growth factors on embryonic development. Growth differentiation factor 9 (GDF9) is one of these growth factors, and it is secreted from the human oocyte during primary follicular development [10]. This factor affects both the initial and the final stages of follicular growth. It is believed that the major biological functions of GDF9 in the ovaries include inducing the mitotic division of granulosa cells to suppress the FSH receptor expression those cells, as well as inducing the expression of kit ligand mRNA in granulosa cells [11]. In these cells, GDF9 is capable of inducing production of inhibin- $\beta$, which exerts paracrine control on inhibin production [12]. GDF9 is also able to stimulate the development of CCs under in vivo conditions and guides the production of hyaluronic acid-rich matrix during CC expansion [13]. It has also been shown that GDF9 is involved in the proliferation of the granulosa cells of the antral follicles prior to ovulation [14].

In recent studies, significant progress has been made in understanding the interaction between CCs and oocytes and the regulation of granulosa cells by oocytes $[15,16]$. Interactions between oocytes and granulosa cells are a way that the ovum controls granulosa cell function both before and after a sudden increase in luteinizing hormone (LH). In antral follicles, GV oocytes are the most notable regulator of CCs [15,17]. High levels of FSH during follicular culture alter the expression of certain compounds in CCs and are also associated with a decrease in anti-Müllerian hormone (AMH) levels [18]. It is believed that, to regulate the level of AMH secreted by the ovary, the levels of GDF9 and bone morphogenetic protein 15 (BMP15) rise, which reflects the response of the ovum to changes occurring in the cells of the cumulus [19]. CCs also play a vital role in meiosis in vitro [20]. One of the most important selection criteria for IVM is the morphology of the oocyte and the condition of the surrounding CCs in the cumulus-oocyte complex (COC), particularly the size and expansion of the CCs. Currently, insufficient information is available regarding the effect of growth factors on the development and quality of human embryos. In this study, we assessed the influence of GDF9 and CCs in IVM culture with human immature oocytes on the rates of maturation and fertilization, embryo formation, and the viability of blastocysts derived from frozen-thawed cleavage embryos.

\section{Methods}

\section{Ovarian hyperstimulation protocol}

Ovarian stimulation was performed using a combination of a gonadotropin-releasing hormone (GnRH) antagonist (Cetrotide; Merck Serono, Darmstadt, Germany) and FSH (Gonal-F; Serono, Geneva, Switzerland). Starting on the second day of the menstrual cycle, 150-225 IU/day of FSH was administered. When a follicle reached a size of 12-14 mm, $\mathrm{GnRH}$ antagonist injections were started, and continued until the follicle reached 17-18 mm. Recombinant human chorionic gonadotropin (Ovitrelle, Merck Serono, Germany) was then administered to trigger final maturation. Approximately 36 hours later, oocyte retrieval was performed using transvaginal ultrasonography-guided aspiration.

\section{GV oocyte collection}

CCs were removed by exposure to $80 \mathrm{IU} / \mathrm{mL}$ hyaluronidase (Sage, Pasadena, CA, USA) for 30 seconds and mechanical pipetting with Pasteur pipettes. After evaluation of the denuded oocytes, the immature oocytes that displayed a vesicular nucleus within the ooplasm and that lacked the first polar body were considered GV oocytes and candidates for IVM.

\section{Preparation of CCs in IVM medium}

This study utilized the cumulus masses of patients in the intracytoplasmic sperm injection (ICSI) program due to male factor infertility, tubal factor infertility, and infertility with unknown etiology. CCs from patients with PCOS, ovarian factor infertility, or endometriosis were excluded. First, the CCs were washed with Ham's F-10 medium containing $10 \%$ human serum albumin (HSA). Then, the cells were washed twice with Ham's F-10 medium, and the suspension concentration was set at 1,000,000 cells $/ \mathrm{mL}$. Then, $20 \mu \mathrm{L}$ of the suspension of CCs and Ham's F-10 medium was dropped. After 30 minutes, the droplet of the suspension was replaced with maturation medium, supplemented with $75 \mathrm{mlU} / \mathrm{mL}$ FSH and $75 \mathrm{mlU} / \mathrm{mL}$ LH (Ferring Pharmaceuticals, Suffern, $\mathrm{NY}, \mathrm{USA}$ ) at $37^{\circ} \mathrm{C}$ and $5 \% \mathrm{CO}_{2}$.

\section{Study design}

A total of $80 \mathrm{GV}$ oocytes were equally assigned to four groups (three experimental and one control) as follows: group I, GV oocytes cultured in droplets of IVM media (control); group II, GV oocytes cultured in IVM medium+CCs; group III, GV oocytes cultured in IVM medium supplemented with $200 \mathrm{ng} / \mathrm{mL}$ GDF9; group IV, GV oocytes 
cultured in droplets of IVM medium+CCs+200 ng/mL GDF9. The oocytes in all groups were incubated under IVM conditions at $37^{\circ} \mathrm{C}$ and $5 \% \mathrm{CO}_{2}$ for 24 hours.

\section{ICSI technique and fertilization assessment}

Each in vitro-matured oocyte was microinjected with a spermatozoon from the patient's husband. Fertilization was evaluated after 16 to 18 hours had elapsed post-ICSI. If two pronuclei were seen, the fertilized oocytes were cultured in fresh cleavage media (G1, Vitrolife, Kungsbacka, Sweden) for 2 days. The embryos were evaluated according to laboratory protocols and the top-quality embryos in the cleavage stage were vitrified.

\section{Cryopreservation and thawing of embryos}

The embryos were cryopreserved using the vitrification method that has been described in existing literature [21]. We used a handmade medium based on Al-Hassani's protocol. First, each embryo was exposed to an equilibration solution consisting of $7.5 \%$ ethylene glycol (EG; Sigma-Aldrich, Steinheim, Germany) and 7.5\% dimethyl sulfoxide (DMSO, Sigma-Aldrich) in Ham's F-10 medium supplemented with 20\% HSA for 5-15 minutes at room temperature. Each embryo was then exposed to a vitrification solution (15\% EG, 15\% DMSO, 0.5 M sucrose; Merck, Darmstadt, Germany) in Ham's F-10 medium supplemented with $20 \% \mathrm{HSA}$ for about 45 to 60 seconds. Next, each embryo was placed on a cryotop sheet and immersed in liquid nitrogen $\left(\mathrm{LN}_{2}\right)$.

To warm the embryos, the Cryotops were removed from the $\mathrm{LN}_{2}$ and placed into a thawing solution (1 M sucrose) for 60 seconds at $37^{\circ} \mathrm{C}$. Then, the embryos were transferred to a dilution solution of 0.5 $\mathrm{M}$ sucrose for 3 minutes, then to another dilution solution of $0.25 \mathrm{M}$ sucrose at room temperature. In the final step, the embryos were transferred into a washing solution and remained there for $5 \mathrm{~min}$ utes. At this time, the embryos were ready to be transferred to the G1 or G2 media, depending on the stage of growth. The embryos were cultured until reaching the blastocyst stage.

\section{Propidium iodide/Hoechst staining}

The blastocysts were rinsed three times in a pre-warmed phosphate-buffered saline (PBS) medium. Then, they were placed in $20 \mu \mathrm{L}$ of propidium iodide ( $\mathrm{P} 4127,300 \mu \mathrm{g} / \mathrm{mL}$ PBS) and Hoechst solution ( $\mathrm{H}$ $33258,5 \mu \mathrm{g} / \mathrm{mL}$ PBS) for 30 minutes in the dark. They were washed several times in PBS and placed in small drops of glycerol. The embryos were viewed with an Olympus BX51 fluorescence microscope (Tokyo, Japan). If the cells appeared blue, they were considered alive; if red, they were considered dead (late apoptotic and necrotic cells).

\section{Statistical analysis}

Data were analyzed using IBM SPSS ver. 21.0 (IBM Corp., Armonk, NY, USA). The similarity between the study groups was assessed using one-way analysis of variance. The chi-square test was used to compare the parameters between the experimental groups. A $p$-value of less than 0.05 was considered to indicate statistical significance.

\section{Results}

\section{The effect of GDF9 and CCs on oocyte maturation rate}

The maturation rate did not differ significantly among the four groups. The highest maturation rate was found in the control group (63.1\%). The maturation rates of both group III (59.1\%) and group IV (57.6\%) were higher than that of group II (50\%) (Figure 1). Degenerated oocytes were not present in significant quantities in any of the four groups.

\section{The effect of GDF9 and CCs on fertilization rate and embryo formation}

The fertilization rate in group II was significantly higher than that in the control group ( $76.5 \%$ vs. $46.2 \%)$. Other groups also had higher fertilization rates than the control group, but those differences were not statistically significant. The frequency of embryo formation was $80.8 \%$ in group II, $88 \%$ in group III, and $84.8 \%$ in group IV; all three groups had significantly higher frequencies of embryo formation than the control group (56.7\%) (Figure 1).

\section{The effect of GDF9 and CCs on blastocyst formation}

Blastocyst formation in the control group did not differ significantly

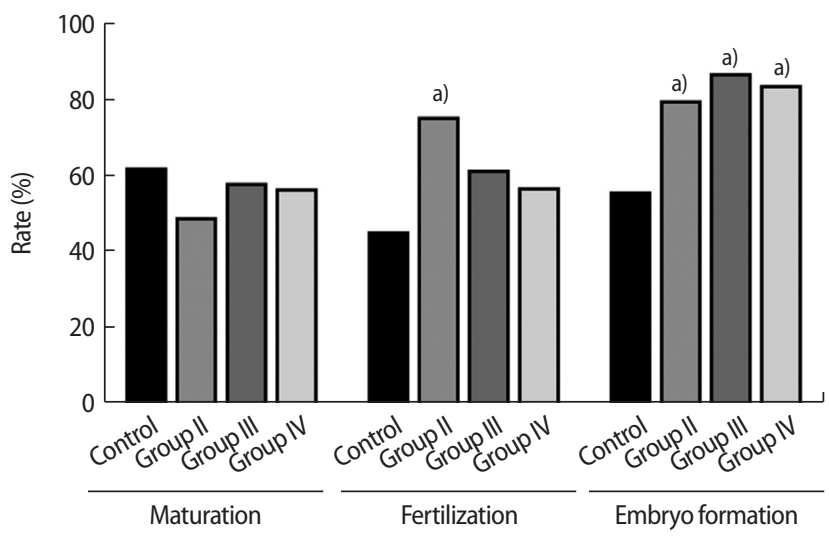

Figure 1. Rates of maturation, fertilization, and embryo formation in the control group (IVM media only), group II (IVM+CCs), group III (IVM+200 ng/mL GDF9), and group IV (IVM+CCs+200 ng/mL GDF9). IVM, in vitro maturation; CC, cumulus cell; GDF9, growth differentiation

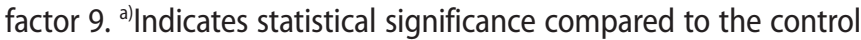
group: $p<0.05$. 

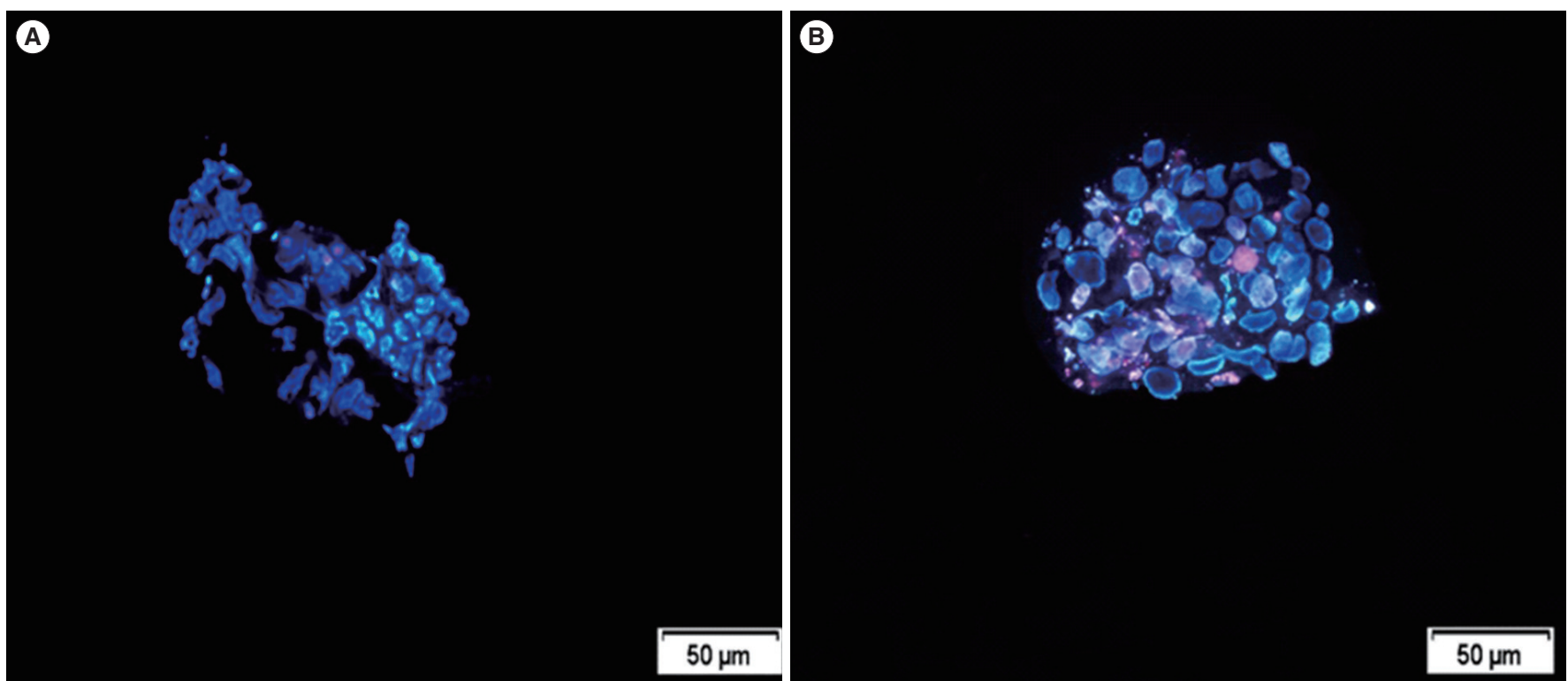

Figure 2. Blastocyst staining with propidium iodide and Hoechst dye. (A) A blastocyst from group Il; the living cells appeared blue and the dead cells appeared red. (B) Blastocyst formation in the control group; the number of cells did not differ significantly between the groups.

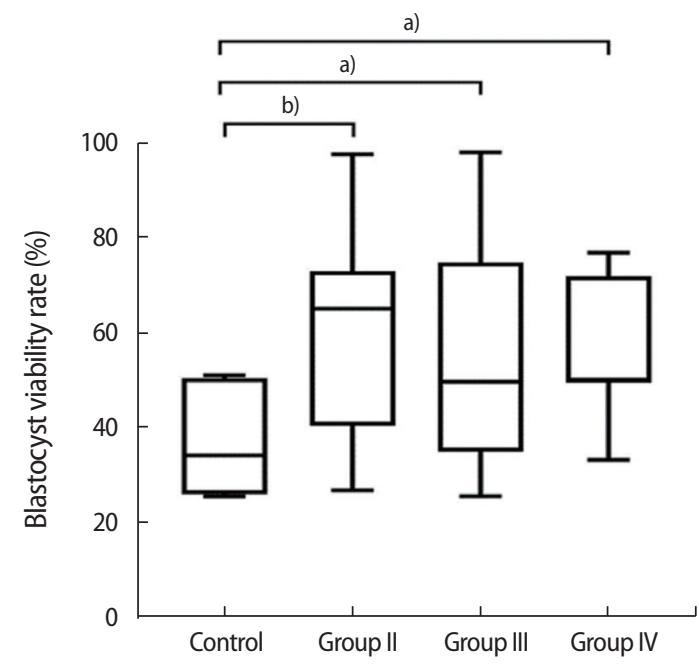

Figure 3. The blastocyst viability rate in the control group (IVM media only), group II (IVM+CCs), group III (IVM+200 ng/mL GDF9), and group IV (IVM+CCs+200 ng/mL GDF9). IVM, in vitro maturation; CC, cumulus cell; GDF9, growth differentiation factor 9. Statistical significance: ${ }^{\text {a) }} p<0.05$; ${ }^{\text {b) }} p<0.01$.

from blastocyst formation in the experimental groups. Additionally, blastocyst formation was not significantly different between groups II and III ( $p=0.86)$, groups II and IV $(p=0.68)$, or groups III and IV $(p=0.98)$ (Figure 2).

\section{The effect of GDF9 and CCs on blastocyst viability}

The mean blastocyst viability rate of group II (58.16\%) was significantly higher than that of the control group $(37.78 \%, p=0.007)$. The mean rate of blastocyst viability in group III (55.91\%) was also significantly higher than that of the control group $(p=0.02)$. Finally, the blastocyst viability rate of group IV $(55.95 \%)$ was again significantly higher than that of the control $(p=0.02)$. No statistically significant differences were found between the blastocyst viability rates of groups II, III, and IV ( $p>0.05$ ). The blastocyst viability rates of the four groups are shown in Figure 3

\section{Discussion}

Based on the results of this study, the maturation and blastocyst formation rates during $\mathrm{COH}$ cycles were not significantly different among the 4 groups under study. However, the use of GDF9 in the IVM culture media did significantly increase the rates of both embryo formation and blastocyst viability. Additionally, CC cells enhanced the rates of fertilization, embryo formation, and viability in blastocysts generated from vitrified cleavage embryos. It has been shown that approximately $15 \%-20 \%$ of the retrieved oocytes in $\mathrm{COH}$ protocols are immature. The use of immature oocytes can be an appealing option for some patients, such as PCOS and cancer patients who are unable to take medicine to stimulate the growth of additional oocytes. Female gamete cryopreservation is an option for these patients, especially in cases involving chemotherapy or radiation [22,23].

Embryos differ in their susceptibility to freezing depending on their stage, from zygote to blastocyst. At each stage of embryonic development, certain proteins and enzymes are made, and the embryo's energy sources change from simple materials, such as pyruvate and lactate, to more complex substances, such as glucose and fatty acids. 
During each cell cycle, the amount of DNA in the embryo increases, and the structure and permeability of the cell membrane, as well as the ultrastructure and thickness of the zona pellucida, change as the embryo develops [24]. It can therefore be concluded that oocytes in different stages display different responses to temperature shocks. The resistance of oocytes to injuries during the freezing process was found to be different in the GV, Ml, and Mll stages due to differences in cellular structure [25]. It has been observed that temperature fluctuations have a direct effect on the cellular and chromosomal organization of Mll oocytes in many species. Complete or relative disorder of microtubules was observed after cooling MII oocytes to room temperature or even lower. Poor embryonic development in a laboratory environment may be an important factor in reduced implantation, and consequently, in reduced pregnancy rates [26].

In previous studies, cryopreservation of single-cell embryos up to the blastocyst stage showed that embryos at the morula stage tolerated the conditions of vitrification. In contrast, two-cell and blastocyst embryos were unable to tolerate the freezing conditions [27,28]. Van der Auwera et al. [27] showed that the concentration and duration of the cryopreservation solution, cell size, membrane permeability, and the stage of embryonic development are important factors that influence the success of vitrification. The vitrification procedure induces ultrastructural alterations, which reduce the competence of cryopreserved oocytes to complete proper maturation. For this reason, it is preferable to first mature the immature oocytes in IVM medium, then vitrify the embryos generated from cleavage of the Mll oocytes [29].

In oocyte maturation, the culture medium influences the number of blastocyst cells. Many attempts have been made to increase the quality of the embryo culture media and bring it closer to the natural conditions of the human reproductive system [7]. The maturation rates of oocytes were evaluated in two different commercial media. The maturation rate, spindle morphometric parameters, and cortical granule density were not statistically significantly different compared to in vivo conditions [30]. The main barriers to expanding the use of these cells are technical problems and the time taken to prepare the single layer of co-culture cells, as well as the probability of transmitting pathogens through these cells to the embryo. CCs are an exception in this regard, as the cells can be taken from the patient and the preparation steps are simple.

In this study, the maturation rate in the control group was higher than that in the other groups. Similarly to our study, Kim et al. [31] reported that the maturation rate of GV oocytes was not affected by the presence or absence of CCs, perhaps because the denuded oocytes were cultured with a single layer of CCs. However, in this situation, direct connection between the oocyte and the CCs is interrupted, and only paracrine effects remain. Reduced and delayed expression of GDF9 and BMP15 during follicular growth is an important fac- tor in the reduction of oocyte maturation in PCOS patients. GDF9 is a member of the large family of $\beta$-growth factors. It plays a role in the growth and development of primary follicles in the ovary, the proliferation of granulosa cells, the rate of ovulation, the cessation of premature ovarian activity, and the differentiation and maturation of oocytes. Evidence supporting the critical role of GDF9 in human fertility has been found in studies exploring the effects of several mutations in GDF9 associated with PCOS. GDF9 has been shown to accumulate in the cytoplasm of oocytes. By upregulating gene expression in the oocyte, it plays an important role in oocyte maturation and CC development [13]. According to the hypothesis derived from this evidence, we expected that embryonic formation would remarkably improve with the addition of GDF9 and CCs to the IVM medium. The results showed that the fertilization rate in group II, as well as embryonic development in all three experimental groups, displayed a significant increase. Additionally, the blastocyst viability rate was significantly higher in the experimental groups than in the control group. In 2014, immature mouse oocytes were evaluated in two different IVM conditions: a control group of oocytes cultured in IVM medium and an experimental group additionally cultured with GDF9 and BMP-15. No improvement in embryonic development was observed to result from the addition of GDF9 and BMP-15 to the IVM media [32]. In a similar study, Su et al. [16] used human GDF9 in IVM with bovine COCs. Their results showed that addition of this factor caused a significant increase in maturation, cleavage, and blastocyst formation, and the number of ICM cells in the group treated with GDF9 was higher than the number in the untreated control group. Additionally, the lowest rate of apoptosis was observed in the GDF9-treated blastocysts. It can be concluded that preparation and improvement of the IVM medium caused an increase in the viability rate of the embryos. Until now, no reports have examined the effect of GDF9 and co-culture with CCs on the formation and viability of human blastocyst cells. Our results showed that application of exogenous GDF9 and co-culture with CCs in IVM improved the viability of the blastocysts. Therefore, exposure of GV oocytes to GDF9 and CCs can potentially increase the chance of fertility.

\section{Conflict of interest}

No potential conflict of interest relevant to this article was reported.

\section{Acknowledgments}

This paper was extracted from MSC thesis of Mahla Honari Chatroudi and financially supported by Shahid Sadoughi University of Medical Sciences, Yazd, Iran. The authors would like to thank the staff of our institute. 


\section{Author contributions}

Conceptualization: MAK, SA. Funding acquisition: MHC. Methodology: MHC, MAK. Project administration: AS, SS. Writing-original draft: MAK. Writing - review \& editing: FA.

\section{References}

1. Faramarzi A, Khalili MA, Ashourzadeh S, Palmerini MG. Does rescue in vitro maturation of germinal vesicle stage oocytes impair embryo morphokinetics development? Zygote 2018;26:430-4.

2. Omidi M, Khalili MA, Ashourzadeh S, Rahimipour M. Zona pellucida birefringence and meiotic spindle visualisation of human oocytes are not influenced by IVM technology. Reprod Fertil Dev 2014;26:407-13.

3. Trounson, A, Gosden R, Eichenlaub-Ritter U. Biology and pathology of the oocyte: role in fertility, medicine and nuclear reprograming. Cambridge: Cambridge University: 2013.

4. Ashourzadeh S, Khalili MA, Omidi M, Mahani SN, Kalantar SM, Aflatoonian $\mathrm{A}$, et al. Noninvasive assays of in vitro matured human oocytes showed insignificant correlation with fertilization and embryo development. Arch Gynecol Obstet 2015;292:459-63.

5. Salustri A, Yanagishita M, Hascall VC. Synthesis and accumulation of hyaluronic acid and proteoglycans in the mouse cumulus celloocyte complex during follicle-stimulating hormone-induced mucification. J Biol Chem 1989;264:13840-7.

6. Swain JE, Carrell D, Cobo A, Meseguer M, Rubio C, Smith GD. Optimizing the culture environment and embryo manipulation to help maintain embryo developmental potential. Fertil Steril 2016;105:571-87.

7. Sanchez F, Lolicato F, Romero S, De Vos M, Van Ranst H, Verheyen $\mathrm{G}$, et al. An improved IVM method for cumulus-oocyte complexes from small follicles in polycystic ovary syndrome patients enhances oocyte competence and embryo yield. Hum Reprod 2017;32:2056-68.

8. Diaz FJ, Wigglesworth K, Eppig JJ. Oocytes determine cumulus cell lineage in mouse ovarian follicles. J Cell Sci 2007;120(Pt 8):1330-40.

9. Zhu XM, Zhu YM, Xu CM, Qian YL, Jin F, Huang HF. Autologous mature follicular fluid: its role in in vitro maturation of human cumulus-removed oocytes. Fertil Steril 2008;90:1094-102.

10. Li Y, Li RQ, Ou SB, Zhang NF, Ren L, Wei LN, et al. Increased GDF9 and BMP15 mRNA levels in cumulus granulosa cells correlate with oocyte maturation, fertilization, and embryo quality in humans. Reprod Biol Endocrinol 2014;12:81.

11. Juengel JL, Hudson NL, Heath DA, Smith P, Reader KL, Lawrence $\mathrm{SB}$, et al. Growth differentiation factor 9 and bone morphoge- netic protein 15 are essential for ovarian follicular development in sheep. Biol Reprod 2002;67:1777-89.

12. Mazerbourg S, Klein C, Roh J, Kaivo-Oja N, Mottershead DG, Korchynskyi $\mathrm{O}$, et al. Growth differentiation factor- 9 signaling is mediated by the type I receptor, activin receptor-like kinase 5 . Mol Endocrinol 2004;18:653-65.

13. Pangas SA, Matzuk MM. The art and artifact of GDF9 activity: Cumulus expansion and the cumulus expansion-enabling factor. Biol Reprod 2005;73:582-5.

14. Belli M, Shimasaki S. Molecular aspects and clinical relevance of GDF9 and BMP15 in ovarian function. Vitam Horm 2018;107: 317-48.

15. Gilchrist RB, Ritter LJ, Myllymaa S, Kaivo-Oja N, Dragovic RA, Hickey TE, et al. Molecular basis of oocyte-paracrine signalling that promotes granulosa cell proliferation. J Cell Sci 2006;119(Pt 18):3811-21.

16. Su YQ, Sugiura K, Eppig JJ. Mouse oocyte control of granulosa cell development and function: paracrine regulation of cumulus cell metabolism. Semin Reprod Med 2009;27:32-42.

17. Gilchrist RB, Ritter LJ, Armstrong DT. Oocyte-somatic cell interactions during follicle development in mammals. Anim Reprod Sci 2004;82-83:431-46.

18. Sugiura K, Pendola FL, Eppig JJ. Oocyte control of metabolic cooperativity between oocytes and companion granulosa cells: energy metabolism. Dev Biol 2005;279:20-30.

19. Sanchez F, Adriaenssens T, Romero S, Smitz J. Different folliclestimulating hormone exposure regimens during antral follicle growth alter gene expression in the cumulus-oocyte complex in mice. Biol Reprod 2010;83:514-24.

20. Vaccari S, Horner K, Mehlmann LM, Conti M. Generation of mouse oocytes defective in CAMP synthesis and degradation: endogenous cyclic AMP is essential for meiotic arrest. Dev Biol 2008;316: 124-34.

21. Al-Hasani S, Ozmen B, Koutlaki N, Schoepper B, Diedrich K, Schultze-Mosgau $A$. Three years of routine vitrification of human zygotes: is it still fair to advocate slow-rate freezing? Reprod Biomed Online 2007;14:288-93.

22. Yazdanpanah F, Khalili MA, Eftekhar M, Karimi H. The effect of vitrification on maturation and viability capacities of immature human oocytes. Arch Gynecol Obstet 2013;288:439-44.

23. Khalili MA, A Nottola S, Shahedi A, Macchiarelli G. Contribution of human oocyte architecture to success of in vitro maturation technology. Iran J Reprod Med 2013;11:1-10.

24. Khalili MA, Maione M, Palmerini MG, Bianchi S, Macchiarelli G, Nottola SA. Ultrastructure of human mature oocytes after vitrification. Eur J Histochem 2012;56:e38.

25. Izadi M, Eftekhar-Vaghefi SH, Akbari H, Asadi-Shekari M, Mokh- 
tari T. Assessment of mouse oocytes ultrastructure following vitrification before and after in vitro maturation. Int J Morphol 2018;36:180-1.

26. Cercas R, Villas C, Pons I, Brana C, Fernandez-Shaw S. Vitrification can modify embryo cleavage stage after warming: should we change endometrial preparation? J Assist Reprod Genet 2012; 29:1363-8.

27. Van der Auwera I, Debrock S, Spiessens C, Afschrift H, Bakelants $E$, Meuleman $C$, et al. A prospective randomized study: day 2 versus day 5 embryo transfer. Hum Reprod 2002;17:1507-12.

28. Hredzak R, Ostro A, Zdilova V, Maracek I, Kacmarik J. Survival of mouse embryos after vitrification depending on the cooling rate of the cryoprotectant solution. Acta Vet Hung 2006;54:117-25.

29. Shahedi A, Hosseini A, Khalili MA, Norouzian M, Salehi M, Piriaei
A, et al. The effect of vitrification on ultrastructure of human in vitro matured germinal vesicle oocytes. Eur J Obstet Gynecol Reprod Biol 2013;167:69-75.

30. Ferrer-Vaquer A, Barragan M, Rodriguez A, Vassena R. Altered cytoplasmic maturation in rescued in vitro matured oocytes. Hum Reprod 2019;34:1095-105

31. Kim BK, Lee SC, Kim KJ, Han CH, Kim JH. In vitro maturation, fertilization, and development of human germinal vesicle oocytes collected from stimulated cycles. Fertil Steril 2000;74:1153-8.

32. Sudiman J, Ritter LJ, Feil DK, Wang X, Chan K, Mottershead DG, et al. Effects of differing oocyte-secreted factors during mouse in vitro maturation on subsequent embryo and fetal development. J Assist Reprod Genet 2014;31:295-306. 